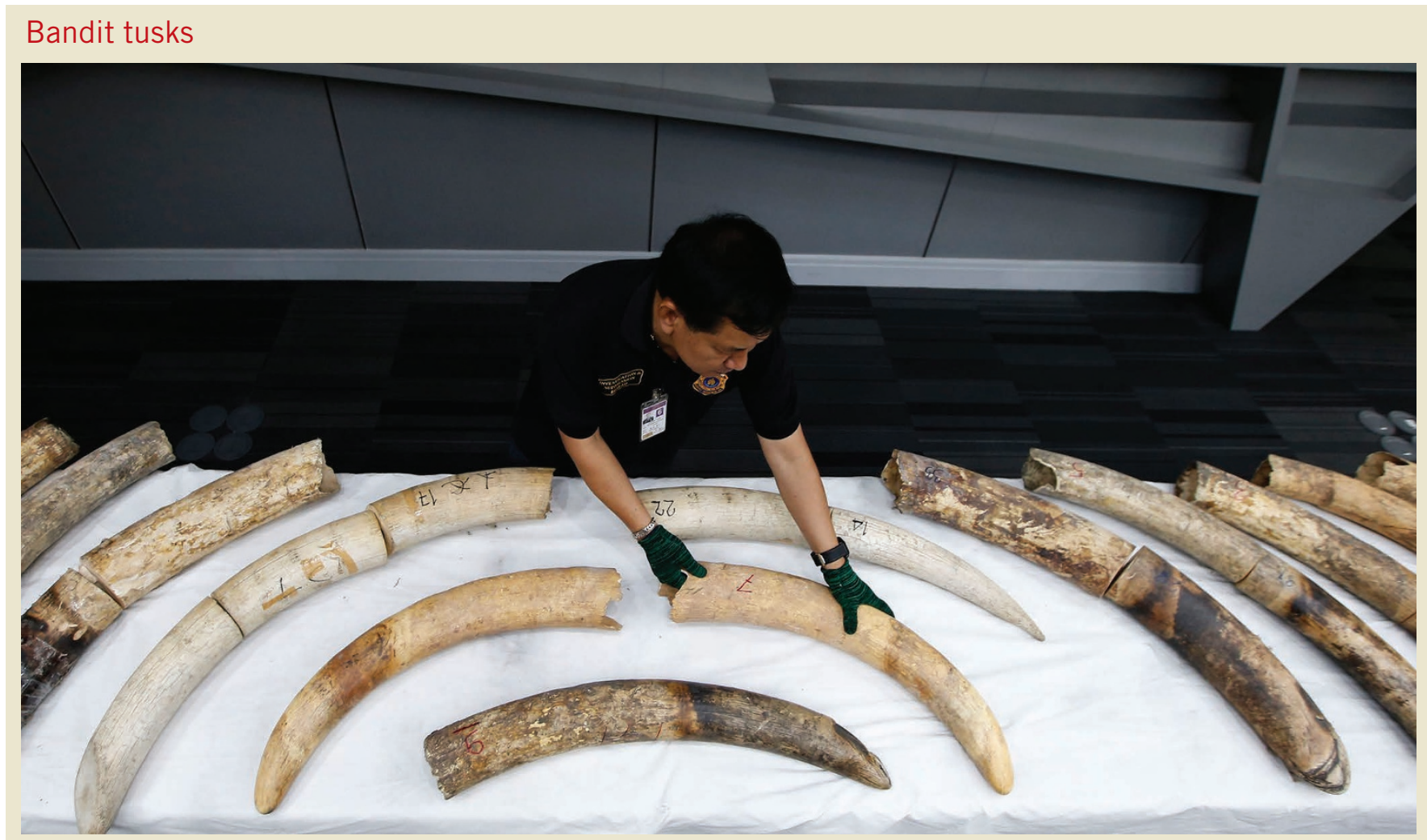

DNA technology is helping customs police track contraband tusks to within kilometers of their place of origin. Researchers at the University of Washington Center for Animal Conservation are mapping tusk DNA to put an end to poaching, a practice that is threatening the African elephant. UW's Sam Wasser devised a technique that can track the origins of tusks to within 300 kilometers by comparing 16 microsatellite DNA patterns against a reference map of elephant genotypes across the continent. This information has enabled law enforcement to locate not just the poachers doing the killing but the cartels that bankroll the operations. The hope is that demonstrating how large these cartels actually are will prompt harsher penalties for the culprits than have been dispensed so far.

\title{
Around the world in a month
}

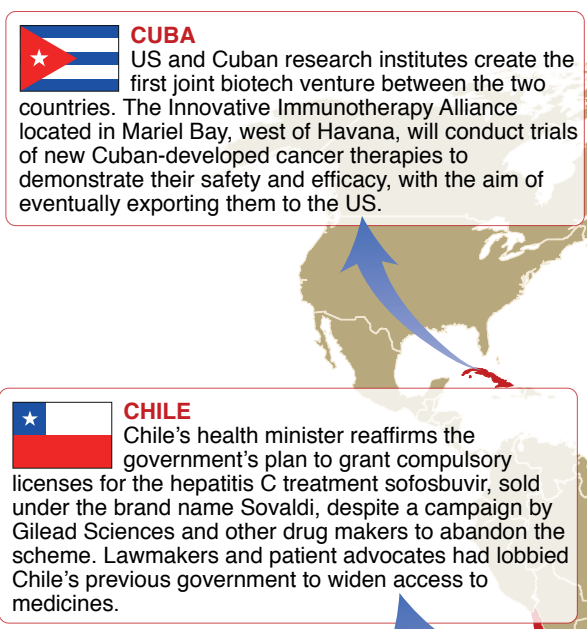
\section{\begin{tabular}{|c|}
\hline$\star \quad$ BURKINA FASO \\
In a first for Africa, the government of \\
Burkina Faso allows researchers to release
\end{tabular}} mosquitoes will not carry any malaria transmissionrelated mutations, but scientists hope the release will increase trust among regulators and locals, in preparation for the future release of 'gene drive' mosquitoes that would significantly reduce the mosquito population.

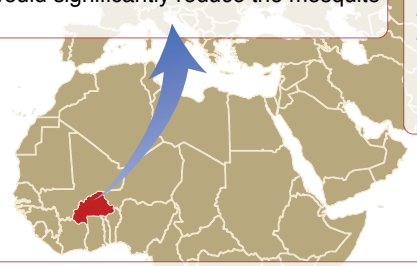

\section{ARGENTINA}

Argentina will commercialize the first drought- and salt-tolerant soybean in 2019 The soybean seeds were developed by splicing a drought-resistance sunflower gene into a soybean seed and have been field tested for three years.

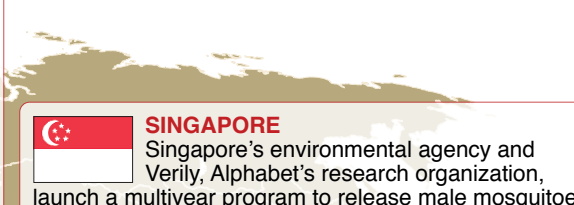
launch a multiyear program to release male mosquitoes that carry Wolbachia, a naturally occurring bacterium that reduces mosquitoes' ability to transmit disease and prevents their eggs from hatching. Verily will use Al to produce, sort and release male mosquitoes with the goal of reducing the overall mosquito population.

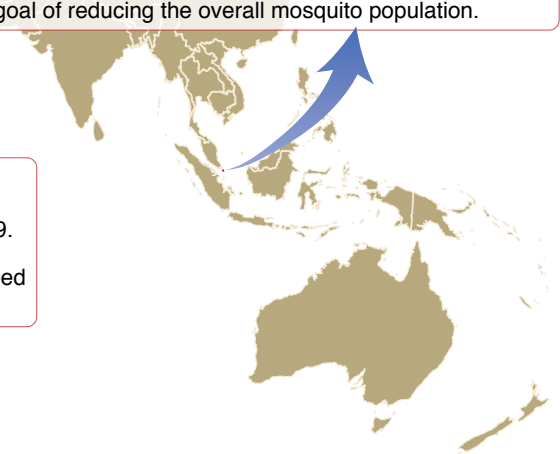

